\title{
MUCOSAL FLAPS STABILISATION IN ENDONASAL DCR
}

\author{
Vikas Sikarwar1, Shivani Tiwari², Akansha Saini ${ }^{3}$
}

1Assistant Professor, Department of ENT, Government Doon Medical College, Dehradun, Uttarakhand, India.

2Senior Resident, Department of ENT, Government Doon Medical College, Dehradun, Uttarakhand, India.

$3 J u n i o r$ Resident, Department of ENT, Government Doon Medical College, Dehradun, Uttarakhand, India.

\section{BACKGROUND}

ABSTRACT

Endo DCR surgical techniques have evolved, giving results comparable to external DCR. Mucosal flaps based DCR has good outcome; still failures are encountered due to unpredicted healing from unstable mucosal flaps causing faulty approximation of these flaps. This aspect of DCR Surgery has to be addressed for better outcome.

The aim of this study was to determine the success rate of endonasal DCR with mucosal flap stabilization

\section{MATERIALS AND METHODS}

This Prospective observational study included 50 patients of chronic Dacryocystitis who underwent DCR surgery. In these patients, DCR was done with our mucosal flaps stabilization technique using Merocel tamponade and results were analysed.

\section{RESULTS}

Authors have utilized this technique in 50 procedures after a minimal follow-up of 6 months. Our success rate is 96 percent.

\section{CONCLUSION}

Mucosal flap stabilization in post-operative period is crucial factor in the success of DCR surgery, our technique of DCR using Merocel Tamponade is simple and effective and it results in better outcome.

\section{KEY WORDS}

Dacryocystitis, DCR, Mucosal Flap Stabilization, Merocel.

HOW TO CITE THIS ARTICLE: Sikarwar V, Tiwari S, Saini A. Mucosal flaps stabilisation in endonasal DCR. J. Evolution Med. Dent. Sci. 2019;8(08):482-483, DOI: 10.14260/jemds/2019/107

\section{BACKGROUND}

Endo DCR techniques are evolving to give results at par with external DCR. Endo DCR Success rates depend on multiple factors like size of bony opening; raw area created, postoperative granulations, adhesions, correct approximation of mucosal flaps. In Post-operative period "Mucosal flap stabilization for desired approximation, is very important factor for final outcome. We introduce a simple yet effective technique in we used Merocel Tamponade for flap stabilization and left them for 72 hours. This simple technique provides effective stabilization of mucosal flaps and improves success rate of DCR.

\section{Aim of The Study}

To estimate success rate of endonasal DCR with mucosal flap stabilization.

\section{MATERIALS AND METHODS}

This Prospective observational study was done from March 2016 to December 2017 at Govt. Doon medical College Dehradun, Uttarakhand. 50 patients of chronic Dacryocystitis who underwent DCR surgery. In these patients DCR was done

'Financial or Other Competing Interest': None.

Submission 29-11-2018, Peer Review 08-02-2019,

Acceptance 16-02-2019, Published 25-02-2019.

Corresponding Author:

Dr. Vikas Sikarwar

Assistant Professor,

Department of ENT,

Government Doon Medical College,

Dehradun, Uttarakhand, India.

E-mail: vikassikarwar79@gmail.com

DOI: $10.14260 /$ jemds/2019/107 with our mucosal flaps stabilization technique using Merocel tamponade. Preoperative workup included Ophthalmologic assessment, sac probing, sac syringing and diagnostic nasal endoscopy. All surgeries were done under General Anaesthesia, success of surgery measured in terms of relief from symptoms i.e. Free from epiphora 3 months after surgery and patency in sac syringing.

\section{Surgical Technique}

Cases were done under general anaesthesia. Nasal cavity was decongested with 1:10, 000 adrenaline and local anaesthetic given with $2 \%$ lignocaine with 1: 80,000 adrenaline. Lateral wall mucosal Incisions were given with 15 no blade, the first incision is made horizontally 8 to $10 \mathrm{~mm}$ above the axilla of middle turbinate and coming forward $10 \mathrm{~mm}$ on frontal process of maxilla then vertical incision is made to about $2 / 3^{\text {rd }}$ of middle turbinates. Mucosal Flap is elevated up to attachment of uncinate process. Chips of lacrimal bone is removed using side knife, then frontal process of maxilla is punched out using Kerrison's bone punch, lacrimal sac is fully exposed, for the exposure of upper of sac is sometimes drilling is required. Then lacrimal probe is then passed through inferior canaliculi causing the tenting of lacrimal sac, incision is given posteriorly creating an anterior based flap. Cuts are given at the upper and lower end of anterior and posterior flaps thereby releasing the tension of flaps and allowing us to marsupialize the entire sac, and then lateral wall mucosal flap is fashioned to create " $\mathrm{C}$ " shaped flap vertical limb of this flap is approximated with posterior lacrimal flap and horizontal limbs are spread over lateral wall to cover the raw surface. These flaps are stabilized by 3 small Merocel pieces: $1^{\text {st }}$ Merocel piece is placed to stabilize posterior lacrimal flap, $2^{\text {nd }}$ Merocel is placed over anterior 
lacrimal flap and $3^{\text {rd }}$ Merocel is used to stabilize the upper limb of lateral wall mucosal flap. Merocel provides firm yet nontraumatic tamponade effect causing effective approximation of mucosal flaps to underlying raw surface and surrounding tissue causing correct approximation of lacrimal and nasal mucosal flaps, hence desired and predicted healing is achieved. These Merocel packs are kept packed for 3 days and Ciplox D Eye drops is started. Packs are removed after 3 days, after which Suction cleaning is done and nasal irrigation started.

\section{RESULTS}

18 males (36\%) and 32 females (64\%) were enrolled, of these 46 (92\%) were primary cases and 4 were revision DCR cases. 20 cases (40\%) had left sided DCR and 30 cases (60\%) had Right sided DCR. Average age of patients was 46 years, youngest patient was 13 years old and oldest was 75 years old. Main symptoms were epiphora (50 cases, $100 \%$ ) and mucocele (8 cases). Septoplasty needed in $40 \%$ cases. Patients were followed up to 6 months to 12 months after surgery, Successful surgical outcome seen in 48 cases (96\%).

\section{DISCUSSION}

Endoscopic dacryocystorhinostomy was first described by Caldwell in the 19th century, [1] but this approach came in to practice in 1990s when endoscopic sinus surgery became popular.[2] In earlier days success rate was lower and varied between $65 \%$ and $90 \%$, which was less than the external DCR where success rate was $90 \%$ to $95 \%$. With better understanding of anatomy of this area and advancements in surgical techniques results improved. Our technique of DCR gives 96 percent success rate.

Success of Endo DCR surgery depends on various factors: a) wide exposure of sac: adequate bone removal is must so entire sac is exposed, [3] drilling of superior part of frontal process of maxilla should be done if required, exposed sac should bulge from lateral wall all around. b) Flap based techniques: Even with wide exposure results are not predictable due to granulation formation and adhesions, which results in Ostia closure. Soon importance of healing by primary intention was realized and various flap-based techniques were developed. The aim of these techniques was to achieve healing by primary intention by covering raw area with mucosal flaps thereby more predicted healing and causing reliable and reproducible results. ${ }^{[4]}$

\section{Stabilisation of Mucosal Flaps}

Mucosal flap-based techniques improved the results but stability of these flaps during healing period is an issue of concern, faulty approximation of flaps results in failure. Nasal to nasal and lacrimal to lacrimal approximation may cause obliteration of lacrimal fistula. Patency depends on the correct approximation of mucosal flaps, to stabilize the flaps various methods were introduced like fashioning of different type of flaps, suturing of flaps, stabilization by clips etc. we used very simple yet effective technique where we used small pieces of Merocel as tamponade for the stabilization of flaps in post-operative period resulting in well mucosalized lacrimal fistula.

Merocel is biocompatible synthetic material with completely open cell structure. It has $100 \%$ open pores in its structure with no dead pockets to hold residues, so it can be left in nose for longer duration without complication like infections and mucosal damage. These properties of Merocel allow us to leave Merocel for longer duration, we find 72 hours is optimum time for flaps to adhered to underlying and surrounding tissue and these mucosal flaps get stabilized resulting in well mucosalized wide lacrimal sac fistula.

Many studies supported the usefulness of Mitomycin C in preventing postoperative adhesions and scarring. ${ }^{[5]}$ We don't use Mitomycin in our DCR cases; covering of raw surfaces by stablized mucosal flaps gives good results.

\section{CONCLUSION}

Flap based techniques of DCR give good long-term results provided these flaps remain stabilized and approximated favourably during healing process. Due importance should be given to stabilization of mucosal flaps. It is one of the most important factors which decides success or failure of surgery. Our technique using Merocel tamponade provides a simple solution for flap stabilization in DCR, gives consistently better and reproducible results.

\section{REFERENCES}

[1] Caldwell GW. Two new operations for obstruction of nasal duct, with preservation of the canaliculi and an incidental description of a new lacrimal probe. Am J Ophthalmol 1893;10:189-93.

[2] Metson R. Endoscopic surgery for lacrimal obstruction. Otolaryngol Head \& Neck Surg 1991;104(4):473-9.

[3] Linberg JV, Anderson RL, Bumsted RM, et al. Study of intranasal ostium external dacryocystorhinostomy. Arch Ophthalmol 1982;100(11):1758-62.

[4] Mueller SK, Freitag SK, Lefebvre DR, et al. Endoscopic DCR using bipedicled interlacing mucosal flaps. Laryngoscope 2018;128(4):794-7.

[5] Zilelioglu G, Ugurbas SH, Anadolu Y, et al. Adjunctive use of mitomycin $\mathrm{C}$ on endoscopic lacrimal surgery. $\mathrm{Br}$ J Opthalmol 1998;82(1):63-6. 